\title{
Novel laser based on magnetic tunneling
}

\author{
A. Kadigrobov ${ }^{1,2,3}$, R.I. Shekhter ${ }^{2}$, and M. Jonson ${ }^{2}$ \\ ${ }^{1}$ Department of Microelectronics and Nanoscience, Chalmers University of Technology \\ S-41296 Göteborg, Sweden \\ E-mail: kadig@tp3.ruhr-uni-bochum.de \\ ${ }^{2}$ Department of Applied Physics, Chalmers University of Technology and Göteborg University \\ SE-41296 Göteborg, Sweden \\ ${ }^{3}$ Theoretische Physik III, Ruhr-Universität Bochum, D-44780 Bochum, Germany
}

Received September 28, 2004

\begin{abstract}
A new principle for a compact spin-based solid-state laser is proposed. It operates in the 1-100 THz regime, which is difficult to reach with small size lasers. Spin-flip processes in ferromagnetic conductors form a basis - the mechanism is due to a coupling of light to the exchange interaction in magnetically ordered conductors via the dependence of the exchange constant on the conduction electron momenta. The interaction strength is proportional to the large exchange energy and exceeds the Zeeman interaction by orders of magnitude. A giant lasing effect is predicted in a system where a population inversion has been created by injection of spin-polarized electrons from one ferromagnetic conductor into another through an intermediate tunnel region or weak link; the magnetizations of the two ferromagnets have different orientations. We show that the laser frequency will be in the range $1-100 \mathrm{THz}$ if the experimental data for ferromagnetic manganese perovskites with nearly $100 \%$ spin polarization are used. The optical gain is estimated to be $g_{\mathrm{opt}} \sim 10^{7} \mathrm{~cm}^{-1}$. This exceeds the gain of conventional semiconductor lasers by 3 or 4 orders of magnitude. An experimental configuration is proposed in order to solve heating problems at a relatively high threshold current density.
\end{abstract}

PACS: 75.70.-i

\section{Introduction}

Conventional electronics, which is based on the manipulation of electronic charge, has been intensively studied for years and has enjoyed wide applications. It will suffice to mention such devices as semiconductor diodes and semiconductor lasers. An appealing alternative is pursued in a new field of solid state physics, the field of «spintronics» [1,2], where one explores the possibility of controlling the spins of conduction electrons and hence to further extend both the area of scientific investigation and the field of applications. Spin-dependent tunneling of electrons has already found commercial applications based on the «giant» magnetoresistance of certain layered structures $[1,2]$. Other applications are bound to follow. In this area magnetically ordered, layered conductors with nearly $100 \%$ spin polarization of the conduction electrons [3] show considerable promise.
A bias voltage applied to a magnetically ordered conductor allows control not only of the energy but also of the spin distribution of electrons that are injected into the magnetic conductor. An example of such a system is presented in Fig. 1, where the hatched region corresponds to an equilibrium distribution of (spin-up) electrons in a spin-polarized conductor. The dotted area marks a nonequilibrium distribution of «hot» (spin-down) electrons. Relaxation of the spindown electrons to an equilibrium configuration requires spin-flip processes and is therefore completely blocked if such processes are not allowed. In the presence of such a «spin-lock» against relaxation, highly excited states in the material may have a long lifetime, which, in turn, may make for novel «spintronics» effects in spin-polarized conductors.

The objective of this paper is to demonstrate how electromagnetic radiation may remove the spin-lock, resulting in a giant lasing effect. A short description 


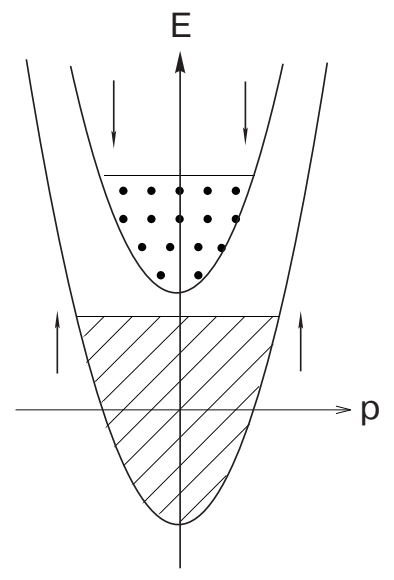

Fig. 1. Schematic representation of the band structure of a magnetically ordered conductor. The hatched region corresponds to an equilibrium distribution of spin-up electrons in the lower band, while the dotted region indicates nonequilibrium distribution of «hot» spin-down electrons in the upper band. Arrows show electron spin directions in the bands.

of this effect has been published as a Letter [4]. It is based on a new mechanism for creating spin-flip processes due to a coupling of the radiation to the exchange interaction in magnetically ordered conductors. This comes about via the dependence of the electron spin-magnetic moment exchange interaction on the moment a of the conduction electrons. As a result, the lasing effect is shown to occur in systems where an inverted electron population has been created by the tunneling injection of spin-polarized electrons from one ferromagnetic conductor to another (the orientation of the magnetization being different in the two ferromagnets). An example of such a system is presented in Fig. 2.

Our estimates show that a laser with an optical gain that exceeds the gain of conventional semiconductor lasers by three or four orders of magnitude can be built, and we argue that laser action can be achieved provided care is taken to design the system so that the active region is not overheated. This is enabled by the high efficiency of the electron spin-flip mechanism described below and the possibility of creating a very high inverted population of electrons in the system under consideration. The frequency of such a laser can be in a wide range that includes the interval $1-100 \mathrm{THz}$, in which attempts to fabricate small size lasers up till now have met severe technical problems [5-10].

In Section 2 we present the above-mentioned spin flip exchange mechanism and find the wave functions of injected electrons in the absence of the electromagnetic field. In Section 3 we calculate the rate of the stimulated photon emission and present estimates of

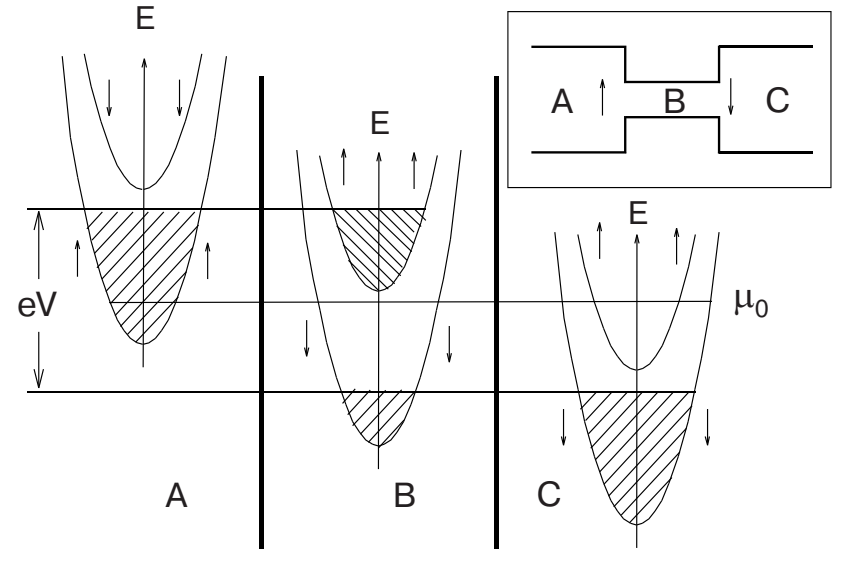

Fig. 2. Schematic illustration of population inversion in a magnetic conductor (region $B$ ). A bias voltage $V$ is applied between magnetic conductors ( $A$ and $C$ ) which have opposite directions of their magnetizations (the thick vertical lines represent potential barriers). Arrows show the electron spin directions in the electron energy bands; $\mu_{0}$ is the equilibrium chemical potential (in the absence of bias). The inset shows a possible realization of the structure in which two magnetic conductors with opposite magnetization directions are coupled through a micro-bridge $(B)$.

the laser optical gain and the threshold current. In the Conclusion we summarize the main results and estimates of the paper, while a cursory description of the laser action is presented in an Appendix for the convenience of the reader.

\section{Population inversion in ferromagnetic conductors and a new mechanism for creating spin-flip processes}

The Hamiltonian for the electrons in a magnetically ordered conductor can be written as

$$
\hat{H}_{0}=\hat{\sigma}_{0} \frac{\hat{p}^{2}}{2 m^{*}}-\hat{\boldsymbol{\sigma}} \mathbf{I}
$$

where $m^{*}$ is the effective mass, $\hat{\mathbf{p}}$ is the momentum operator, $\hat{\boldsymbol{\sigma}}$ are Pauli matrices, $\sigma_{0}$ is the $2 \times 2$ unity matrix, and $\mathbf{I}$ is the exchange energy. According to Eq. (1) the dispersion law for electrons with spins up/down is

$$
E_{\uparrow \downarrow}(p)=\frac{p^{2}}{2 m^{*}} \mp I .
$$

We will deal with a system, schematically presented in Fig. 2, in which two potential barriers divide the magnetic conductor into three parts; the magnetization of two adjacent magnetic conductors (regions $A$ and $B$ ) are pointing in opposite directions. A bias voltage $V$ is applied between regions $A$ and $C$. We assume 
that the spin relaxation time $v_{s}^{-1}$ [11], the time $t_{E}$ of electron energy relaxation without changing spin direction, and the electron tunneling time $t_{\text {tun }}$ obey the double inequality $t_{E} \ll t_{\text {tun }} \ll v_{S}^{-1}$. In the absence of spin-flip processes, the energy relaxation of injected spinpolarized electrons creates a nonequilibrium state in which equilibrium is established only inside each group of electrons with a fixed spinpolarization. Therefore, in region $B$ electrons inside the spin-up and spin-down energy bands are in local equilibria described by the different chemical potentials $\mu \uparrow$ and $\mu_{\downarrow}$, while the system as a whole is far from equilibrium.

According to Eq. (2) the energy conservation law for vertical transition of electrons with emission of photons of frequency $\omega$ does not depend on the electron momentum:

$$
\hbar \omega=E_{\downarrow}(p)-E_{\uparrow}(p)=2 I .
$$

It follows that for $\omega=2 I / \hbar$ all hot» electrons are in resonance with the electromagnetic field, and hence stimulated emission of light due to transitions of electrons from filled states in the upper band to empty states in the lower band is possible for all electrons in an energy range $\mu_{\uparrow}-\mu_{\downarrow}$. As is seen from Fig. 2, the population inversion needed for lasing requires a bias voltage $V>2 I / e$ ( $e$ is the electron charge).

The conventional Zeeman term $\hat{H}_{Z}=\left(g \mu_{B} / 2\right) \mathbf{H} \hat{\boldsymbol{\sigma}}$ describing interaction between the (hot) electrons and an electromagnetic field does provide a mechanism for stimulated radiative transitions between the energy bands containing electrons with opposite spin directions. However, it is relatively small in magnitude and it is not the most important mechanism. For ferromagnets, we would like to suggest a much more effective mechanism of interaction between light and conduction electron spins. This mechanism is based on the dependence of the exchange energy $\mathbf{I}$ on the momentum $\mathbf{p}$ of the conduction electron. The momentum dependence has to do with the overlap of the wave functions of the conduction electron and the magnetic subsystem (see, e.g., [12]). It is determined by the value of $p a / \hbar$, where $a$ is the characteristic size of the orbital (that is, one may estimate $\partial I / \partial p \sim(a / \hbar) I$, where $a$ is an atomic-scale length). That is why it varies with the momentum of the conduction electron. In the absence of an electromagnetic field the Hamiltonian that describes this situation can be written as

$$
\varepsilon(\hat{\mathbf{p}})=\hat{\sigma_{0}} \frac{\hat{\mathbf{p}}^{2}}{2 m^{*}}-\hat{\boldsymbol{\sigma}} \cdot \mathbf{I}(\hat{\mathbf{p}}) .
$$

In the presence of an electromagnetic field described by a vector potential $\mathbf{A}$, the momentum operator $\hat{\mathbf{p}}$ in Eq. (4) must be changed to $\hat{\mathbf{p}}-(e / c) \mathbf{A}$ ( $c$ is the light velocity), and one obtains an effective Hamiltonian $\hat{H}_{\text {eff }}=\varepsilon(\hat{\mathbf{p}}-(e / c) \mathbf{A})-\hat{\boldsymbol{\sigma}} \mathbf{I}(\hat{\mathbf{p}}-(e / c) \mathbf{A})$. Expanding in powers of $(e / c) \mathbf{A}$, one gets an effective Hamiltonian of the form

$$
\hat{H}_{\mathrm{eff}}=\varepsilon(\hat{\mathbf{p}})+\hat{H}_{\mathrm{eff}}^{(1)},
$$

where the perturbation Hamiltonian [13] is

$$
\hat{H}_{\mathrm{eff}}^{(1)}=-\frac{e}{2 c} \hat{\boldsymbol{\sigma}}\left(A_{i} \frac{\partial \mathbf{I}}{\partial p_{i}}+\frac{\partial \mathbf{I}}{\partial p_{i}} A_{i}\right)_{\mathbf{p}=\hat{\mathbf{p}}} .
$$

In Eq. (6) we have omitted the term

$$
\hat{H}_{\mathrm{eff}}^{(1)}=\hat{\boldsymbol{\sigma}}_{0} \frac{e}{m^{*} c} \mathbf{A} \cdot \hat{\mathbf{p}},
$$

which does not flip spins; summation over repeated indices is implied: $a_{i} b_{i} \equiv \mathbf{a b}$.

If the injected electrons are prepared in such a way that their spins are not parallel to the magnetization in the active region $B$ (see Fig. 2), the Hamiltonian (6) produces spin-flips and hence stimulates the needed radiative transitions of hot electrons in the upper band to the lower energy band. This process is illustrated in Fig. 3, where an electron (with its spin parallel to the magnetization) is impinging on the boundary from the left, passes through the boundary and is scattered into a quantum superposition of spin-up and spin-down states in the active region $B$ to the right of the boundary.

In order to find the probability amplitude for a radiative transition of an electron caused by perturbation Eq. (6) we obtain the zero-approximation wave function of the electron as a solution of the Schrödinger equation $\varepsilon(\hat{\mathbf{p}})|\Psi\rangle=E|\Psi\rangle$, neglecting the dependence of the exchange interaction $\mathbf{I}$ on $\hat{\mathbf{p}}$. Taking into account the fact that the electron wave-function is a two-component spinor,

$$
\Psi(\mathbf{r})=\left(\begin{array}{l}
\Psi_{1} \\
\Psi_{2}
\end{array}\right),
$$

and using Eq. (4), one can write the Schrödinger equation as

$$
\begin{aligned}
& \left(\frac{\hat{\mathbf{p}}^{2}}{2 m^{*}}-E-I_{z}(x)\right) \Psi_{1}-I_{+}^{*}(x) \Psi_{2}=0 \\
& \left(\frac{\hat{\mathbf{p}}^{2}}{2 m^{*}}-E+I_{z}(x)\right) \Psi_{2}-I_{+}(x) \Psi_{1}=0 .
\end{aligned}
$$

Here the $x$ axis is perpendicular and the $y$ and $z$ axes parallel to the boundary (see Fig. 3), and we assume that in the left-side ferromagnet $(x<0)$ the direction of $\mathbf{I}$ is along the $z$ axis (i.e., $\mathbf{I}=(0,0, I))$ while in the right-side ferromagnet $(x>0)$ its direction is differ- 


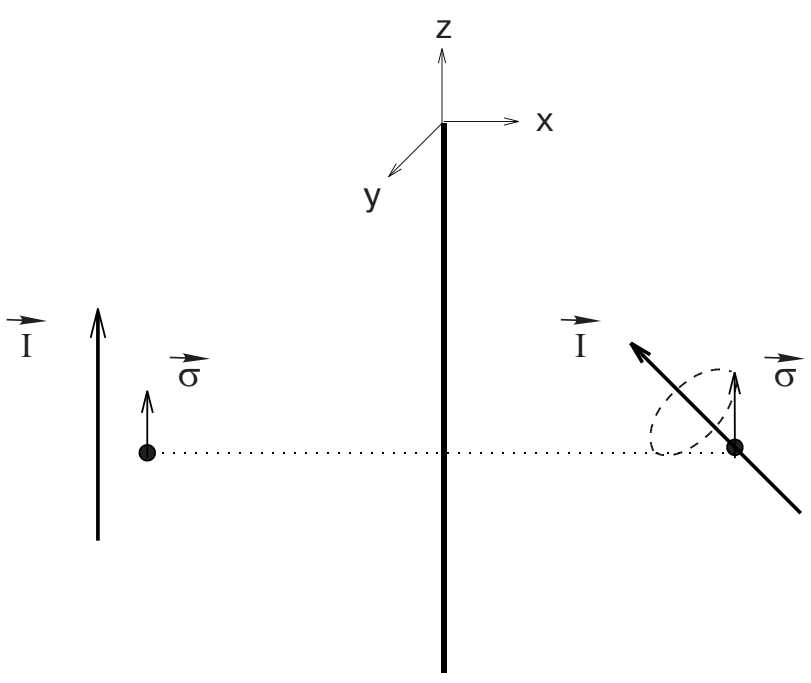

Fig. 3. Schematic illustration of how electrons are injected into the active region for the case that the adjacent ferromagnets have different magnetization directions (shown with long arrows; electron spins, $\sigma$, are shown with short arrows with black dots at the end). An electron from the left-side ferromagnet with its spin parallel to the magnetization direction passes through a sharp boundary (shown as a vertical thick line) between the ferromagnets without changing spin direction. In the right-side ferromagnet it emerges with its spin in a superposition of the spin-up and spin-down states. The classical precession of the spin is indicated by a dashed-line ellipse.

ent and all three components of $\mathbf{I}$ are present: $\mathbf{I}=$ $=\left(I_{x}, I_{y}, I_{z}\right), I_{x}^{2}+I_{y}^{2}+I_{z}^{2}=I^{2}$, and $I_{+}=I_{x}+i I_{y}$. We also assume that the width of the intermediate layer between the left and right ferromagnets $d$ (the width of the «domain wall») satisfies the inequality $d<\left\langle\hbar v_{F} / I\left(v_{F}\right.\right.$ is the electron Fermi velocity). In this case an electron passes this region without changing spin direction and hence one may solve Eq. (9) considering $\mathbf{I}$ to be constant in the injecting region (left-hand side of Fig. 3) and in the active region (the right-hand side of Fig. 3) and matching the wave functions at the boundary $x=0$.

Considering the case of an electron incident from the injection region with its spin parallel to the magnetization direction, one readily finds the wave function of the electron in the injection region $(x<0)$ to be

$$
\begin{array}{r}
\Psi^{(l)}(\mathbf{r})=\exp \left(i \mathbf{p}_{\perp} \mathbf{r}_{\perp}\right)\left\{\left(\begin{array}{l}
1 \\
0
\end{array}\right) \exp \left(i p_{1} x\right)+\right. \\
\left.+a_{1}^{(l)}\left(\begin{array}{l}
1 \\
0
\end{array}\right) \exp \left(-i p_{1} x\right)+a_{2}^{(l)}\left(\begin{array}{l}
1 \\
0
\end{array}\right) \exp \left(-i p_{2} x\right)\right\},
\end{array}
$$

while in the active region $(x>0)$ the wave function is

$$
\begin{gathered}
\Psi^{(r)}(\mathbf{r})=\exp \left(i \mathbf{p}_{\perp} \mathbf{r}_{\perp}\right) \times \\
\times\left\{a_{1}^{(r)}\left(\begin{array}{c}
1 \\
I_{+} /\left(I_{z}+I\right)
\end{array}\right) \exp \left(i p_{1} x\right)+\right. \\
\left.+a_{2}^{(r)}\left(\begin{array}{c}
1 \\
I_{+} /\left(I_{z}-I\right)
\end{array}\right) \exp \left(i p_{2} x\right)\right\} .
\end{gathered}
$$

Here the projections of the electron momentum and the coordinate on the boundary are denoted by $\mathbf{p}_{\perp}=\left(0, p_{y}, p_{z}\right) \quad$ and $\quad \mathbf{r}_{\perp}=(0, y, z)$, while $p_{1,2}=$ $=\sqrt{2 m^{*}(E \pm I)-p_{\perp}^{2}}$. Matching the wave functions (10) and (11), one finds the coefficients $a_{(1,2)}^{(l, r)}$ to be

$$
\begin{aligned}
a_{1}^{(l)} & =\frac{p_{1}\left(p_{2}-p_{1}\right)}{\left(p_{1}+p_{2}\right)^{2}-\left(p_{1}-p_{2}\right)^{2} \cos ^{2} \varphi / 2}\left(\frac{I_{+}}{\left|I_{+}\right|} \sin \varphi\right), \\
a_{2}^{(l)} & =\frac{p_{2}^{2}-p_{1}^{2}}{\left(p_{1}+p_{2}\right)^{2}-\left(p_{1}-p_{2}\right)^{2} \cos ^{2} \varphi / 2}\left(\frac{I_{+}}{\left|I_{+}\right|} \sin ^{2} \varphi / 2\right)
\end{aligned}
$$

and

$$
\begin{aligned}
& a_{1}^{(r)}=\frac{4 p_{1} p_{2}}{\left(p_{1}+p_{2}\right)^{2}-\left(p_{1}-p_{2}\right)^{2} \cos ^{2} \varphi / 2} \cos ^{2} \varphi / 2, \\
& a_{2}^{(r)}=\frac{2 p_{1}\left(p_{1}+p_{2}\right)}{\left(p_{1}+p_{2}\right)^{2}-\left(p_{1}-p_{2}\right)^{2} \cos ^{2} \varphi / 2} \sin ^{2} \varphi / 2 .
\end{aligned}
$$

\section{Stimulated emission rate and giant laser effect in ferromagnetic conductors}

The characteristics of lasers can be described by rate equations which are obtained by considering the time evolution of the number of photons and the number of nonequilibrium electrons in the active region (see, e.g., [16,17]). The simplest form of the set of rate equations for the density of photons $N_{p}$ and the density of electrons $N_{\uparrow}$ in the upper band can be written as

$$
\left\{\begin{array}{l}
\dot{N}_{p}=G\left(N_{\uparrow}\right) N_{p}-v_{p} N_{p} \\
\dot{N}_{\uparrow}=\eta J / e V_{0}-v_{s} N_{\uparrow}-G\left(N_{\uparrow}\right) N_{p}
\end{array}\right.
$$

(See Section 3.3.6 in Ref. 17). Here $G\left(N_{\uparrow}\right)$ and the stimulated emission rate $R_{\mathrm{st}}$ are related, $R_{\mathrm{st}}=$ $=G\left(N_{\uparrow}\right) N_{p} ; v_{p}$ is the photon relaxation rate; $J$ is the injection current; $\eta$ is the internal efficiency: the fraction of the injection current that generates electrons in the upper band of the active region; $V_{0}$ is the volume of the active region.

With the electron wave functions defined we can find the rate $R_{\text {st }}$ of radiative electronic transitions by assuming the vector potential $\mathbf{A}(\mathbf{r}, t)$ in Eq. (6) to be a 
plane wave of the form $A_{0} \exp i(-\mathbf{k r}+\omega t)$ and using Fermi's Golden Rule:

$$
\begin{aligned}
R_{\text {st }} & =\frac{2 \pi}{\hbar} \int\left|H_{21}\right|^{2}\left[f_{2}\left(1-f_{1}\right)-f_{1}\left(1-f_{2}\right)\right] \times \\
& \times \delta\left(E_{2}(\mathbf{p})-E_{1}(\mathbf{p})-\hbar \omega\right) \frac{d \mathbf{p}}{(2 \pi \hbar)^{3}} .
\end{aligned}
$$

Here

$$
H_{21} \equiv\left\langle\Psi_{f}\left|\hat{H}_{\mathrm{eff}}^{(1)}\right| \Psi_{i}\right\rangle=\int \Psi_{f}^{*}(\mathbf{r}) \hat{H}_{\mathrm{eff}}^{(1)} \Psi_{i}(\mathbf{r}) d^{3} \mathbf{r}
$$

is the matrix element corresponding to the transition from the initial state $\Psi_{i}(\mathbf{r})$ (which belongs to the initial energy $E_{i}$ in the upper band) to the final state $\Psi_{f}(\mathbf{r})$ (which belongs to the final energy $E_{f}$ in the lower band); functions $f_{1,2}$ are the Fermi functions of electrons in the upper and lower bands:

$$
f_{1,2}=\frac{1}{\exp \left(E-\mu_{\uparrow, \downarrow}\right) / k T+1} .
$$

Using Eq. (6) and Eq. (11), one sees that the matrix element $\left\langle\Psi_{f}\left|\hat{H}_{\text {eff }}^{(1)}\right| \Psi_{i}\right\rangle$ (the probability amplitude for a radiative electron transition between the unperturbed energy bands) is non-zero if $p_{1}\left(E_{i}\right)=p_{2}\left(E_{f}\right)$. From here it follows that the difference between the initial and the final energies should be

$$
E_{i}(\mathbf{p})-E_{f}(\mathbf{p})=2 I(\mathbf{p}) .
$$

As the dependence of $I(\mathrm{p})$ on the electron momentum $\mathbf{p}$ is relatively weak, the dependence of the argument of the $\delta$-function in Eq. (15) on $\mathbf{p}$ is also weak. This can result in a divergent integral under the resonance condition $\hbar \omega=p_{1}\left(E_{i}\right)-p_{2}\left(E_{f}\right)=2 I(\mathbf{p})$. As is often the case, the amplitude of the resonance is cut off by the finite electron lifetime in a given state. We assume the energy $\delta$-function to be broadened due to the spin-flip processes and rewrite Eq. (15) as

$$
\begin{gathered}
R_{\mathrm{st}}=\frac{2 \pi}{\hbar} \int\left|H_{21}\right|^{2}\left(f_{2}-f_{1}\right) \times \\
\times \frac{\pi^{-1} \hbar v_{s}}{(2 I(\mathbf{p})-\hbar \omega)^{2}+\left(\hbar v_{s}\right)^{2}} \frac{d \mathbf{p}}{(2 \pi \hbar)^{3}} .
\end{gathered}
$$

Taking the wave functions of Eq. (11) as the initial and final states and performing standard calculations using Eqs. (6), (16), and (18), one readily finds the stimulated transition rate.

i) Under the assumption that $\left|I^{\prime}\right| p_{F \uparrow}<\hbar v_{S}\left(p_{F \uparrow}\right.$ is the Fermi momentum of electrons in the upper band), that is the additional dispersion caused by the dependence of $I$ on the electron momentum (see Eq. (4)) is smaller than the broadening of the electron energy due to spin-flip processes, the stimulated transition rate per unit volume of active material is

$$
\begin{aligned}
& R_{\mathrm{st}}=\frac{32 \pi \mathrm{e}^{2} \mu}{n^{2}} \frac{\left|\mathbf{I}^{\prime}\right|^{2}}{\omega} \frac{\hbar v_{s}\left(b_{1} N_{\uparrow}-b_{2} N_{\downarrow}\right)}{(\hbar \omega-2 I)^{2}+\left(\hbar v_{s}\right)^{2}} N_{p} \times \\
& \times\left\{\left(\left[\mathbf{\epsilon}_{a}\left[\mathbf{\epsilon}_{\mathrm{dr}} \cdot \mathbf{\epsilon}_{a}\right]\right] \cdot \mathbf{\epsilon}_{i}\right)^{2}+\left(\left[\mathbf{\epsilon}_{\mathrm{dr}} \cdot \mathbf{\epsilon}_{a}\right] \cdot \mathbf{\epsilon}_{i}\right)^{2}\right\}
\end{aligned}
$$

Here $\mu$ and $n$ are the magnetic permeability and the refractive index of the medium, respectively, $N_{\uparrow}$ and $N_{\downarrow}$ are the densities of electrons with spin up and down, $N_{p}$ is the photon density, $\omega$ is the photon frequency, the constants $b_{1}$ and $b_{2}$ are of order unity, the unit vectors $\mathbf{\epsilon}_{a}$ and $\mathbf{\epsilon}_{i}$ are directed along the magnetizations in the active (right-hand side of Fig. 3) and injection (left-hand side of Fig. 3) regions, respectively, while $\mathbf{\epsilon}_{\mathrm{dr}}$ is parallel to the vector $\mathbf{I}^{\prime} \equiv e_{i} \partial \mathbf{I} / \partial p_{i}$, where $\mathbf{e}$ is the unit polarization vector in the direction of the vector potential $\mathbf{A}$.

ii) In the opposite limit, $\left|I^{\prime}\right| p_{F \uparrow}>\hbar_{s}$ one has

$$
\begin{array}{r}
\quad R_{\mathrm{st}}=\frac{32 \pi}{\hbar \omega} \frac{\mathrm{e}^{2} \mu}{n^{2}}\left|\mathbf{I}^{\prime}\right|^{2}\left(b_{3} N_{\uparrow}^{2 / 3}-b_{4} N_{\downarrow}^{2 / 3}\right) N_{p} \times \\
\times\left\{\left(\left[\mathbf{\epsilon}_{a}\left[\mathbf{\epsilon}_{\mathrm{dr}} \cdot \mathbf{\epsilon}_{a}\right]\right] \cdot \mathbf{\epsilon}_{i}\right)^{2}+\left(\left[\mathbf{\epsilon}_{\mathrm{dr}} \cdot \mathbf{\epsilon}_{a}\right] \cdot \mathbf{\epsilon}_{i}\right)^{2}\right\},
\end{array}
$$

where the constants $b_{3,4} \sim 1$.

As is seen from the set of rate equations Eq. (14) (see also the Appendix), one of the necessary conditions for the lasing effect to be realized is

$$
R_{\mathrm{st}}=v_{p} N_{p} .
$$

We consider the case when damping of electromagnetic waves is mainly due to absorption by free charge carriers, the frequency of the photon relaxation being $v_{p}=2 k \omega / n$ [18]. For estimating the parameters of the problem we use standard formulae for the refractive index $n$ and the absorption coefficient $k$ for metals subject to electromagnetic fields [19],

$$
v_{p}=\frac{\mu}{n^{2}} \frac{4 \pi \sigma(0)}{1+\left(\omega t_{0}\right)^{2}},
$$

where $\sigma(0)$ is the static conductivity of the conductor and $t_{0}$ is the transport electron relaxation time.

Using Eq. (19), Eq. (22), and the estimate $\left|I^{\prime}\right| \sim I / p_{0}$, one can rewrite Eq. (21) as

$$
\frac{\left(N_{\uparrow}-N_{\downarrow}\right)}{N_{\uparrow}+N_{\downarrow}} \approx \frac{\hbar v_{s}}{2 I} \frac{p_{0}^{2} / m^{*}}{\hbar t_{0}^{-1}} \frac{1}{1+\left(\omega t_{0}\right)^{2}},
$$

where $p_{0}=\hbar / a \sim 10^{-19} \mathrm{erg} \cdot \mathrm{s} / \mathrm{cm}$.

It seems that to achieve the lasing effect the most favorable materials are ferromagnetic manganese perovskites with nearly $100 \%$ spin polarization of the conduction electrons $[3,20,21]$. The high degree of polarization of the carriers permits the creation of a pop- 
ulation inversion of the energy bands in the active region $B$ (see Fig. 2). Here and below we use experimental values of the needed parameters: the mean free path $l_{0}=1.4 \cdot 10^{-7} \mathrm{~cm}$, the Fermi velocity $v_{F} \approx 10^{8}$ $\mathrm{cm} / \mathrm{s}, t_{0} \approx 10^{-15} \mathrm{~s}$, and $m^{*}=0.3 m_{e}$, where $m_{e}$ is the free electron mass, the number of carriers $\approx 3.4 \cdot 10^{21}$, the resistivity $\rho \sim 10^{-4}-10^{-3} \Omega \cdot \mathrm{cm}$ [22]. Inserting these values into Eq. (23), one finds this lasing condition to be

$$
\frac{N_{\uparrow}-N_{\downarrow}}{N_{\uparrow}+N_{\downarrow}} \approx 5 \frac{\hbar v_{s}}{I} .
$$

For the case $\left|I^{\prime}\right| p_{F \uparrow} \gg \hbar v_{S}$ (see Eq. (20)), the lasing condition Eq. (21) is

$$
\frac{N_{\uparrow}^{2 / 3}-N_{\downarrow}^{2 / 3}}{N_{\uparrow}+N_{\downarrow}} \approx 0.5 \cdot 10^{7} \mathrm{~cm}^{-1} .
$$

It follows from these equations that the lasing condition $R_{\mathrm{st}}=v_{s} N_{p}$ is easily satisfied, since one needs $\hbar v_{s} / I$ to be less than $10^{-1}$, while theoretical estimate of the spin relaxation rate $v_{s}$ gives the value $10^{-2}$ for this ratio. Estimations based on the above experimental values of the parameters show the optical gain to be $g_{\text {opt }}=(n / c) R_{\text {st }} \sim 10^{7} \mathrm{~cm}^{-1}$ and the threshold current density $j_{\text {th }}=e l v_{S} N_{\uparrow} \sim 10^{7}-10^{8} \mathrm{~A} / \mathrm{cm}^{2}$ for the length of the active region $l=10^{-5} \mathrm{~cm}$. Estimations for $N_{e} \sim 10^{18} \mathrm{~cm}^{-3}$ show the optical gain and the threshold current to be $g_{\mathrm{opt}} \sim 10^{3}-10^{4} \mathrm{~cm}^{-1}$ and $j_{\text {th }} \sim 10^{5} \mathrm{~A} / \mathrm{cm}^{2}$.

We predict an extremely large optical gain in systems with a high density of charge carriers. The price to be paid for the gain exceeding what can be achieved in semiconductors by 3 or 4 orders of magnitude is the high currents needed for an effective tunneling pumping of the system. The current value $j=10^{6}-10^{8} \mathrm{~A} / \mathrm{cm}^{2}$ seems to be very large for homogeneous bulk metals because of the accompanying Joule heating. Special measures are needed to avoid heating the active, lasing region. One solution to that problem is to arrange for the current injection to be inhomogeneous in space. This can be achieved if the magnetic conductors are electrically connected through an electric point contact. In such systems the spreading of the current far from the narrow point contact provides for an efficient dissipation of heat [24]. A current density $j \sim 10^{8} \mathrm{~A} / \mathrm{cm}^{2}$ can be reached without significant heating of the contact region $[24,25]$. On the other hand, the extremely large optical gain $g_{\mathrm{opt}} \sim 10^{7} \mathrm{~cm}^{-1}$ means that it is enough to have a small volume of active lasing region. Such a structure can be prepared on the basis of the technique suggested in Ref. 26 for fabrication of biepitaxial films of $\mathrm{La}_{0.7} \mathrm{Sr}_{0.3} \mathrm{MnO}_{3}$ with $45^{\circ}$ in-plane rotated domains. In this case the sug- gested laser is a series of point contacts in a thin film of the ferromagnetic metal with the resonator cavity above it.

\section{Conclusion}

We have proposed a new principle for a compact solid-state laser working in the $1-100 \mathrm{THz}$ regime. The proposed laser is based on a new mechanism for creating spin-flip processes in ferromagnetic conductors. The mechanism is due to the interaction of light with conduction electrons; the interaction strength, being proportional to the large exchange energy, exceeds the Zeeman interaction by orders of magnitude. On the basis of this interaction, a giant lasing effect was predicted for systems where a population inversion can be created by tunneling injection of spin-polarized electrons from one ferromagnetic conductor to another - the magnetization of the two ferromagnets having different orientations. Using experimental data for ferromagnetic manganese perovskites with nearly $100 \%$ spin polarization we showed the laser frequency to be in the range 1-100 THz. The optical gain was estimated to be of order $10^{7} \mathrm{~cm}^{-1}$, which exceeds the gain of conventional semiconductor lasers by 3 or 4 orders of magnitude. An experimental study based on a point contact geometry to avoid heating by the necessarily large injection currents was proposed and discussed.

We thank L.Y. Gorelik, V. Kozub, G.D. Mahan, and R. Gunnarsson for helpful discussions. We are also grateful to T. Claeson for fruitful discussions and critical reading of the manuscript. A.K. acknowledges the hospitality of the Theoretische Physik III Institut at the Ruhr-Universität, Bochum, Germany.

A.K. gratefully acknowledes financial support from the Royal Swedish Academy of Sciences (KVA) and SFB 491. M.J and R.S. acknowledge the financial support from the NANODEV SSF center and from the SSF programme on Magnetoelectronic Nanodevice Physics.

\section{Appendix}

The set of differential nonlinear equations (14) allows two types of steady-state solutions (see, e.g. $[16,17])$ :

$$
N_{P}=0 ; \quad N_{\uparrow}^{(0)}=\frac{\eta J}{e V_{0} v_{s}}
$$

and

$$
G\left(N_{\uparrow}^{(\text {las })}\right)=v_{p} ; \quad N_{p}^{\text {(las) }}=\frac{\eta J / e V_{0}-v_{s} N_{\uparrow}^{(\text {las })}}{v_{p}}
$$




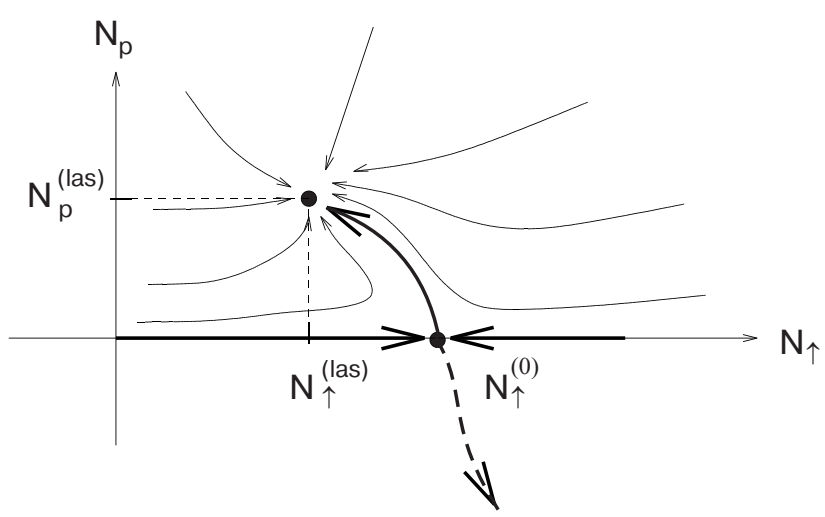

Fig. 4. Schematic presentation of the phase plane of Eq. (14); thick dots show the stable fix point $\left(N_{p}^{\text {(las) }}\right.$, $\left.N_{\uparrow}^{(\text {las) }}\right)$ and the saddle point $\left(N_{p}^{(0)}, N_{\uparrow}^{(0)}\right)$; thick arrows are separaterices and thin arrows show trajectories starting from various initial points; all of them end up at the stable point $\left(N_{p}^{(\text {las })}, N_{\uparrow}^{\text {(las) }}\right)$, which corresponds to the stationary state of the laser.

(for the case under consideration, the explicit forms of $G\left(N_{\uparrow}\right)=R_{\text {st }} / N_{p}$ are easily found from Eq. (19) and Eq. (20)).

The steady-state solution Eq. (24) exists for any value of the injection current, while the steady-state solution Eq. $(25)$ is nonphysical $\left(N_{p}^{(\text {las })}<0\right)$ until the value of the injection current exceeds the threshold $J^{\text {(th) }}$ at which the right-hand side of the second equation in Eq. (25) becomes positive. As follows from Eq. (25) the value of the threshold current is

$$
J^{(\text {th })}=\frac{e v_{s} V_{0} N_{\uparrow}^{(\text {las })}}{\eta} .
$$

The steady-state solution (24) describes a state of the system in which the lasing effect is absent: photons are not emitted and the number of electrons in the upper band is controlled exclusively by the injection current and the nonradiative relaxation $v_{s}$. One can see from Eq. (14) that this state is stable in the range of injection currents $J<J^{\text {(th) }}$.

When the strength of the injection current exceeds the threshold $\left(J>J^{(\text {th })}\right)$ this nonradiative state becomes unstable, and the system spontaneously switches to a new stablestate which is described by Eq. (25): the population of electrons in the upper band is clamped to the threshold value $N_{\uparrow}^{(\text {las })}$ while the number of photons in the system increases with an increase of the injection current. Figure 4 schematically shows the phase plane $\left(N_{p}(t), N_{\uparrow}(t)\right)$ of Eq. (14) for the case $J>J^{\text {(th) }}$. The thick dots are the stable fixed point $\left(N_{p}^{(\text {las })}, N_{\uparrow}^{\text {(las) }}\right.$ ) and the saddle point $\left(N_{p}^{(0)}, N_{\uparrow}^{(0)}\right)$, whose separatrices are shown by thick lines (the separatrix lying in the nonphysical part of the plane is shown by a thick dashed line); thin lines in the figure represent trajectories of the system which start from various initial states and finish in the stable fixed point $\left(N_{p}^{\text {(las) }}, N_{\uparrow}^{\text {(las) }}\right)$; arrows show the direction of motion along the trajectories.

1. G.A. Prinz, Science 282, 1660 (1998).

2. S.A. Wolf, D.D. Awschalom, R.A. Buhrman, J.M. Daughton, S. von Molnar, M.L. Roukes, A.Y. Chtchelkanova, and D.M. Treger, Science 294, 1488 (2001).

3. Colossal Magnetoresistive Oxides, Yoshinori Tokura (ed.), Gordon and Breach Science Publishers, Amsterdam (2000).

4. A Kadigrobov, Z. Ivanov, T. Claeson, R.I. Shekhter, and M. Jonson, Europhys. Lett., 67, 948 (2004).

5. R. Kohler, A. Tredicucci, F. Beltram, H. Beere, E. Linfeld, A. Giles Devies, D.A. Ritchle, R.C. Lotti, and F. Rossi, Nature 417, 156 (2002).

6. S.G. Pavlov, R.Kh. Zhukavin, E.E. Orlova, V.N. Shastin, A.V. Kirsavin, H.-W. Hübers, K. Auen, and H. Riemann, Phys. Rev. Lett. 84, 5220 (2000).

7. E. Bründermann, D.R. Chamberlin, and E.E. Haller, Appl. Phys. Lett. 76, 2991 (2000).

8. D.M. Matsuura, M. Tani, and K. Sakai, Appl. Phys. Lett. 70, 559 (1997).

9. R. Kersting, K. Unterreiner, G. Strasser, H.F. Kauffmann, and E. Gornik, Phys. Rev. Lett. 79, 3038 (1997).

10. B.B. Hu, X.-C. Zhang, and D.H. Auston, Phys. Rev. Lett. 67, 2709 (1991).

11. The main mechanism for relaxation of hot electrons is the spin-flip process caused by emission of spin-waves.

12. H.J. Zeiger and G.W. Pratt, Magnetic Interactions in Solids, Clarendon Press, Oxford (1973); S.V. Vonsovskii, Magnetism, v. 2, Wisley, New York (1974).

13. The Hamiltonian Eq. (6) gives an effective spin-orbit interaction in magnetic films which are usually under a strong strain caused by the substrate and grain boundaries (see, e.g., [14,15] and references there). If $p a / \hbar<<1$, for bulk materials $\partial \mathbf{I} / \partial p_{i}=0$, one should take the next term in the expansion, that is change $\partial \mathbf{I} / \partial p_{i}$ to $p_{k} \partial^{2} \mathbf{I} / \partial p_{k} \partial p_{i}$ in Eq. (6).

14. Yan Wu, Y. Suzuki, U. Rudiger, J Yu, and A.D. Kent, Appl. Phys. Lett. 74, 2295 (1999).

15. Yeong-Ah Soh, P.G. Evans, Z. Cai, B. Lai, C.-Y. Kim, G. Aeppli, N.D. Mathur, M.G. Blamire, and E.D. Isacs, J. Appl. Phys. 91, 7742 (2002).

16. G.P. Agrawal and N.K. Dutta, Long-Wavelength Semiconductor Lasers, Van Nostrand Reinhold Company, New York (1986).

17. G.P. Agrawal, Fiber Optic Communication System, Wiley New York (1997).

18. F.J. Blatt, Physics of Electronic Conduction in Solids, McGraw-Hill Book Company (1968).

19. J.M. Ziman, Principles of the Theory of Solids, Cambrige (1964).

20. J.-H. Park, E. Vescovo, H.-J. Kim, C. Kwon, R. Ramesh, and T. Venkatesan, Nature 392, 794 (1998). 
21. Y.-D. Chuang, A.D. Gromko, D.S. Dessau, T. Kimura, and Y. Tokura, Science 292, 1509 (2001).

22. The values are taken for a compound similiar to the perovskite manganites found to be nearly $100 \%$ spinpolarized, see [21].

23. S.J. Lloid, N.D. Mathur, J.C. Loudon, and P.A. Midgley, Phys. Rev. B64, 172407 (2001).

24. A.V. Khotkevich and I.K. Yanson, Atlas of Point Contact Spectra of Electron-Phonon Interactions in
Metals, Kluwer Academic Publishers, Boston/Dordrecht/London (1995).

25. J.J. Versluijs, M.A. Bari, and M.D. Coey, Phys. Rev. Lett. 87, 026601 (2001).

26. R. Mathieu, P. Svedlindh, R.A. Chakalov, and Z.G. Ivanov, Phys. Rev. B62, 3333 (2000). 